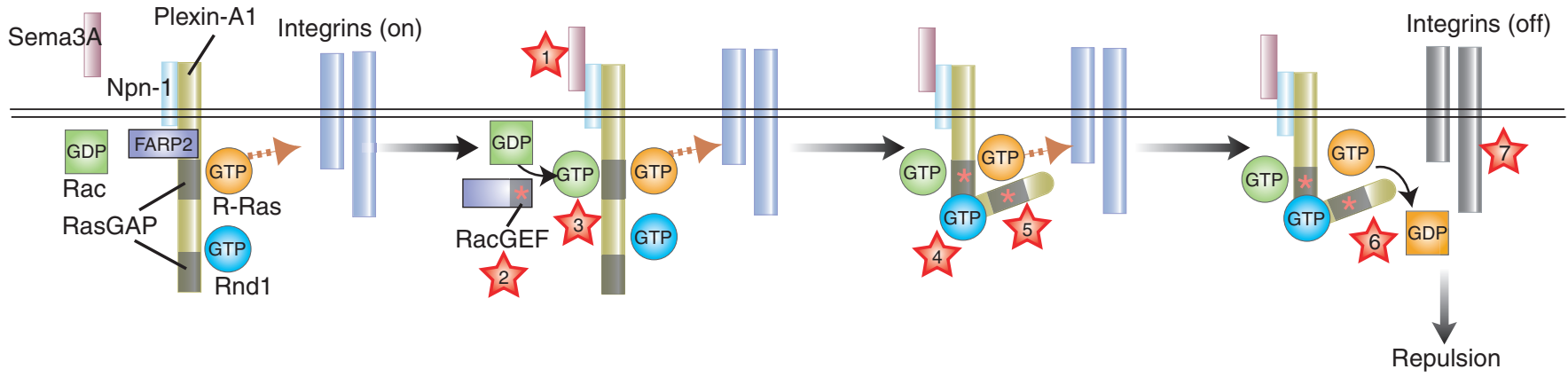

Figure 1 A model for plexin-A1 activation and growth-cone repulsion by semaphorin-3A. Binding of semaphorin-3A (Sema3A) to neuropilin-1 (Npn-1; step 1) triggers FARP2 dissociation from plexin-A1 and activates FARP2's RacGEF (step 2), leading to activation of Rac (step 3). Activation of Rac stimulates binding of Rnd1 to plexin-A1 (step 4), which activates plexin-A1's latent RasGAP (step 5). The plexin-A1 RasGAP downregulates R-Ras (step 6), leading to inhibition of integrin function (step 7) and growth-cone repulsion. Further details of the model are described in the text.

FARP2 with PIPKI $\gamma 661$, a phosphatidylinositol kinase that has been implicated in promoting the assembly of integrin-containing focal complexes $^{12,13}$. Semaphorin-3A stimulation reduces PIPKI 6661 kinase activity, providing a second possible way for semaphorin-3A to inhibit integrin function. However, the significance of PIPKI $\gamma 661$ in growth-cone guidance remains uncertain, as PIPKI $\gamma 661$ knockdown had no detectable effect on repulsion. Nonetheless, these results are especially intriguing because MICALs - a family of plexin-A1-binding proteins that, like FARP2, are involved in semaphorin-3A-mediated repulsion ${ }^{14}$ _interact with CasL, a focal adhesion protein involved in integrin-mediated motility. The route from the semaphorin-3A receptor complex to integrins could turn out to be well traveled.

The model for semaphorin-3A receptor activation that emerges from this work raises important questions to address in future studies. First, how does Rac activation contribute to the binding of Rnd 1 to
plexin-A1? Rac could interact directly with plexin-A1 to induce a conformational change needed for Rnd1 binding or, alternatively, could act more indirectly. Second, how does Rnd 1 binding to plexin-A1 stimulate plexin-Al's RasGAP activity? Because the Rnd1 binding site is located between the two regions that comprise the plexin-A1 RasGAP domain, one obvious model is that Rnd 1 binding causes a conformational change in plexin-A1 that promotes RasGAP activity. Future biochemical and structural studies will undoubtedly clarify these issues. In addition, what are the critical molecular links between the semaphorin-3A receptor and integrins? It will be important to determine how inhibition of R-Ras decreases integrin function, and which aspects of semaphorin-3A-mediated repulsion involve changes in integrin signaling. Finally, the path for semaphorin-3A receptor activation uncovered by Toyofuku $e t$ al. suggests several molecules, such as FARP2, that could serve as targets through which other signaling pathways could modulate a growth cone's response to semaphorin-3A. The semaphorin-3A guidance receptor is shaping up to be a sophisticated signaling machine.

1. Pasterkamp, R.J. \& Kolodkin, A.L. Curr. Opin Neurobiol. 13, 79-89 (2003).

2. Toyofuku, T. et al. Nat. Neurosci. 8, 1712-1719 (2005).

3. Luo, L. Nat. Rev. Neurosci. 1, 173-180 (2000).

4. Oinuma, I., Ishikawa, Y., Katoh, H. \& Negishi, M. Science 305, 862-865 (2004).

5. Zanata, S.M., Hovatta, I., Rohm, B. \& Püschel, A.W. J. Neurosci. 22, 471-477 (2002).

6. Turner, L.J., Nicholls, S. \& Hall, A. J. Biol. Chem. 279 33199-33205 (2004).

7. Jin, Z. \& Strittmatter, S.M. J. Neurosci. 17, 62566263 (1997).

8. Kuhn, T.B., Brown, M.D., Wilcox, C.L., Raper, J.A. \& Bamburg, J.R. J. Neurosci. 19, 1965-1975 (1999).

9. Kubo, T. et al. J. Neurosci. 22, 8504-8513 (2002).

10. Zhang, Z., Vuori, K., Wang, H., Reed, J.C. \& Ruoslahti, E. Cel/ 85, 61-69 (1996).

11. Serini, G. et al. Nature 424, 391-397 (2003).

12. Ling, K., Doughman, R.L., Firestone, A.J., Bunce, M.W. \& Anderson, R.A. Nature 420, 89-93 (2002).

13. Di Paolo, G. et al. Nature 420, 85-89 (2002).

14. Terman, J.R., Mao, T., Pasterkamp, R.J., Yu, H.H. \& Kolodkin, A.L. Cell 109, 887-900 (2002)

\title{
Did you feel that?
}

How does subjective experience correlate with neural activity? Activity in early sensory cortex reflects accuracy in discriminating different stimuli, and is thought to be key in determining perceptual judgments. Now a study on page 1698 of this issue shows that in monkeys judging stimuli at the threshold of detection, activity in frontal rather than sensory cortex correlates with subjective experience.

Lafuente and Romo recorded single-neuron responses from primary somatosensory and medial premotor cortex in monkeys trained to report the presence or absence of a mechanical vibration applied to their fingertips. The strength of the vibration varied, and it was sometimes just at the threshold of detection, so that the monkeys made mistakes. Activity in the somatosensory cortex accurately reflected stimulus strength, but it did not correlate with the monkeys' perceptual reports. Medial premotor cortex activity was independent of stimulus strength, but covaried with the monkeys' subjective judgments, even when they gave an incorrect response. Electrically stimulating the medial premotor

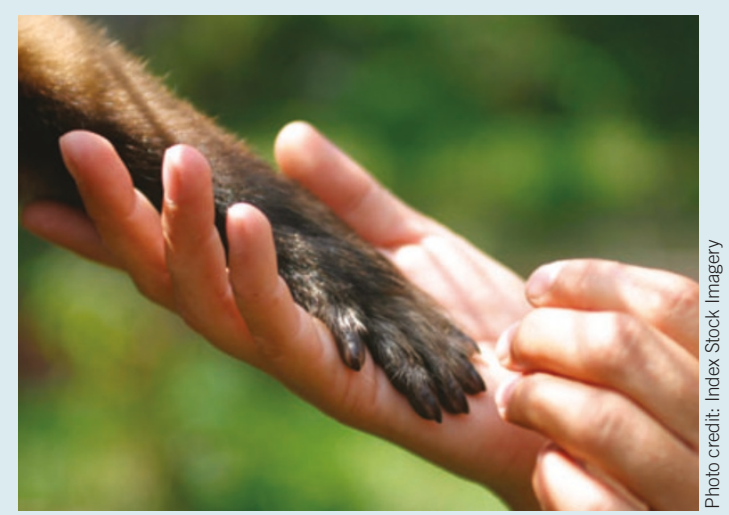
cortex caused the monkeys to respond almost exactly as they did to real mechanical stimulation. These results suggest that somatosensory cortex represents the physical properties of a stimulus, whereas frontal neurons are more involved in subjective perceptual judgments.

Charvy Narain 